\title{
Unified Neural Network Based Pathologic Event Reconstruction Using Spatial Heart Model
}

\author{
Sándor M. Szilágyi ${ }^{1}$, László Szilágyi ${ }^{1,2}$, Attila Frigy ${ }^{3}$, Levente K. Görög ${ }^{1}$, \\ and Zoltán Benyó ${ }^{2}$ \\ ${ }^{1}$ Sapientia - Hungarian Science University of Transylvania, \\ Faculty of Technical and Human Science, Târgu-Mureş, Romania \\ szs@ms.sapientia.ro \\ ${ }^{2}$ Budapest University of Technology and Economics, \\ Dept. of Control Engineering and Information Technology, Budapest, Hungary \\ ${ }^{3}$ County Medical Clinic No. 4, Târgu-Mureş, Romania
}

\begin{abstract}
This paper presents a new way to solve the inverse problem of electrocardiography in terms of heart model parameters. The developed event estimation and recognition method uses a unified neural network (UNN)-based optimization system to determine the most relevant heart model parameters. A UNN-based preliminary ECG analyzer system has been created to reduce the searching space of the optimization algorithm. The optimal model parameters were determined by a relation between objective function minimization and robustness of the solution. The final evaluation results, validated by physicians, were about $96 \%$ correct. Starting from the fact that input ECGs contained various malfunction cases, such as Wolff-Parkinson-White (WPW) syndrome, atrial and ventricular fibrillation, these results suggest this approach provides a robust inverse solution, circumventing most of the difficulties of the ECG inverse problem.
\end{abstract}

Keywords: Heart model, unified neural network, inverse ECG problem.

\section{Introduction}

Nowadays the health problems related to the malfunction of the heart affects large groups of people and have become the most important mortality factor 3]. These malfunctions are usually caused by heart attack, rhythm disturbances and pathological degenerations. Modern health study is focusing on predicting these kinds of tragic events, and identifying the endangered patients, to make it possible to apply a preventing therapy.

Traditional computerized electrocardiogram (ECG) analyzer systems used the collected signal as an input to suggest an empiric-information-based evaluation of the ECG [10]. These systems may recognize various waveforms, but the leakage of information about the inner functioning of the heart inhibits to understand the producing phenomena 13 .

The construction of a heart model 20] may allow computers to recognize the origin and the evolvement process of the ECG signal [5]. These systems may 
unify the vast empiric information applied in traditional systems with modelbased-recognition, creating hybrid processing structures [17]. These hybrid systems may activate the model-based-approach at any moment to handle correctly almost all unrecognizable waveform. The strange waveforms may appear in case of unknown patients or uncommon states, such as ventricular fibrillation [19. In these cases the model-based approach estimates the causes of the encountered phenomenon.

A dynamic organ such as the heart places special demands on modeling techniques. To understand its physiology and patho-physiology, not only the electrical activity and spatial distribution of its structures is important, but also their movement during cardiac cycles [10]. The shape of the measured ECG signal is influenced during repolarization by the mechanical contraction of the heart 9 .

The main problem of inverse ECG processing consists in reconstruction of cardiac electrical events from measurements [14]. In contrast to the forward problem of electrocardiography, the inverse problem does not possess a mathematically unique solution [6] and in order to improve stability, it needs to adopt regularization techniques 415 .

The problem of multiple solutions of the inverse models enforced the development of several approaches such as equivalent cardiac generators (such as equivalent dipole and multi-pole) 9], heart surface isochrones [1, or epicardial potential [2. These methods led to a significant progress, but the different uncertainty elements of the processing method hinder the potentially beneficial ECG inverse solutions from becoming a routine clinical tool.

An almost complete ECG data acquisition from the human torso is accomplished by the body surface potential mapping (BSPM) technique [8]. BSPM may have a great advantage over the standard 12-lead system in different situations due to deeper accessible information. Mirvis has shown some cases of BSPM recordings that clearly demonstrate the inadequacies of the standard ECG lead sets in a variety of pathologies [8]. The better understanding of the depolarization-repolarization mechanism may enlighten the origin of diverse pathological events.

In the area of data processing, numerous interesting biomedical applications of artificial neural networks are included [7]. The best known neural solutions involve multilayer perceptrons [20], Kohonen self-organizing networks [17], fuzzy or neuro-fuzzy systems [16], genetic algorithms [19] and the combination of various solutions within a hybrid system [1].

Earlier heart modeling systems applied many neural networks and chose the best one, while the others were discarded. After a deep investigation of the obtained results, it was recognized that the most efficient approaches should rely on the combination of many classifiers utilizing either different classifier network structures or different data preprocessing methods [1].

The conventional artificial neural networks (ANNs) suffer from diverse drawbacks that can be handled by the support vector machines (SVMs) pioneered by Vapnik [21], which had to face to following problems: 
- Modern biological problems are high-dimensional, and if the underlying mapping is not very smooth, the linear paradigm needs an exponentially increasing number of terms with an increasing dimensionality of the input space, that implies an increase in the number of independent variables. This is known as 'the curse of dimensionality';

- The real-life data generation laws may typically be far from the normal distribution and a model-builder must handle any kind of distribution in order to construct an efficient learning algorithm;

- The maximum likelihood estimator (consequently the sum-of-error-squares cost function, too) should be replaced by a new induction paradigm that is uniformly better. This indicator may accomplish the properly modeling of non-Gaussian distributions.

SVM classifiers have become quite popular due to their robustness and stability [12. A SVM used in a heart modeling system is rigorously based on statistical learning theory and simultaneously minimizes the training and test errors. Apart from that, they produce a unique globally optimal solution and hence are extensively used in diverse applications including medical diagnosis [16].

This paper presents an event recognition study performed with ECG signal analysis and 3D heart model using unified neural networks (UNNs). These UNNs are based both on conventional ANNs and SVMs. The main purpose is to evaluate the strength and weakness of the method, and to analyze the cooperation efficiency in malfunction diagnosis.

\section{Materials and Methods}

\subsection{Unified Neural Networks}

In case of two-class classification using linear discriminant functions, the respective decision hypersurface in the $n$-dimensional feature space is a hyperplane that can be described as:

$$
g(x)=w^{T} \cdot x+w_{0}=0,
$$

where $w=\left[w_{1}, \ldots, w_{n}\right]^{T}$ is known as the weight vector and $w_{0}$ as the threshold value. A given vector $x_{d}$ is situated on the decision hyperplane if only if $g\left(x_{d}\right)=$ 0 . The distance $z$ between a vector $x$ and the decision hyperplane is computed as: $z=|g(x)| /\|w\|$, where $\|w\|=\sqrt{\sum_{i=1}^{n} w_{i}^{2}}$.

The purpose in a classification problem consists in the optimization of a vector $w$ in such a way, that the criteria function $J(w)$ is minimized. Let $\omega_{1}$ and $\omega_{2}$ be the two classes that we need to separate. We assume this task can be performed using a linear relation, meaning that there exists at least one such hyperplane $\bar{w}$ that fulfils the following relations:

$$
\bar{w}^{T} \cdot x>0 \text { for } \forall x \in \omega_{1} \text {, and } \bar{w}^{T} \cdot x<0 \text { for } \forall x \in \omega_{2} .
$$

If we design a classifier with a desired output $y=1$ for $\forall x \in \omega_{1}$ and $y=-1$ for $\forall x \in \omega_{2}$, and try to modify weights in vector $w$ in such a way that the 
criteria function $J(w)=\sum_{i=1}^{N}\left(y_{i}-f_{s}\left(w^{T} \cdot x_{i}\right)\right)^{2}$ is minimized, then we have constructed a root mean square (RMS) error based separation method. In the previous formula, $f_{s}$ denotes a sigmoid function.

The SVM-s based classifier algorithms are very popular due their robustness. The main concept incorporates the search for the 'most robust solution' vector $w$ that gives the maximum possible margin. The margin is represented by the minimal distance $z=|g(x)| /|| w||$, which requires the minimization of $\|w\|$.

Although both these methods, the traditional RMSE approximation and SVM-based classification, deliver good results in a certain noise-free environment, in biomedical simulation such sterile conditions never occur, mainly because of measurement errors and the improper estimation of unmeasurable biological parameters.

Root mean square classifiers have the following drawbacks:

- Improper solution in case of asymmetric transfer functions;

- Large estimation error of the criteria function in case of border-close high dispersion (uncertain) inputs;

- In a noisy environment, the criteria function may possess multiple local minimal solutions that may cause low quality results;

- The white noise is 'non-learnable', so the function $J(w)$ will saturate at an uncontrollable error level.

The SVM produces a considerably superior result in hostile environment, and can avoid the above mentioned problems, but fails to take into consideration the topology of the input vectors, as presented in Fig. 1(a). This topology becomes more important is case of multi-dimensional spaces and non-linear separation borders, than in case of linear separation in a two dimensional space.

To overcome the above mentioned problems for both presented classification metods, we propose for classification a UNN. The main difference between UNN and the described classifiers consists in the equation of its criteria function:

$$
J(w)=\lambda_{d} \cdot \sum_{i=1}^{N}\left(y_{i}-f_{s}\left(w^{T} \cdot x_{i}\right)\right)^{2}+\lambda_{m} \cdot f_{m}(z)+\lambda_{u} \cdot \sum_{i=1}^{N} f_{\mathrm{u}}\left(w^{T} \cdot x_{i}\right),
$$

that is composed by three additive terms responsible for the difference error, margin and smoothness, respectively. The function $f_{u}(\alpha)=\alpha^{-2}$ represents a repelling force that doesn't let the boundary be close to any of the vectors $x_{i}$. Coefficients $\lambda_{d}, \lambda_{m}, \lambda_{u}$ adjust the tradeoff among these three terms. The margin value is represented by $z$.

The presented UNN can work in non-linear environment, too. In this case the distance of a point from the separation hyperstructure is considered the closest distance from any point of it. The SVM formulation can work in the same manner, the structure tries to have a shape that keeps maximal distance from the clusters elements. The third term forces the separation structure to places that suffer from minimal repelling force.

The non-linear form of the criteria function takes into consideration the topology of the separation hyperstructure as presented in Figure 1(b). For example, 


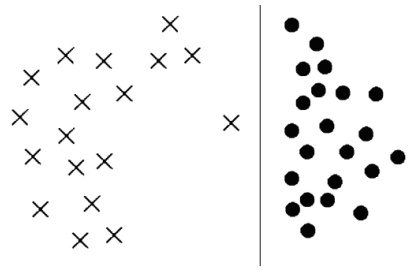

(a)

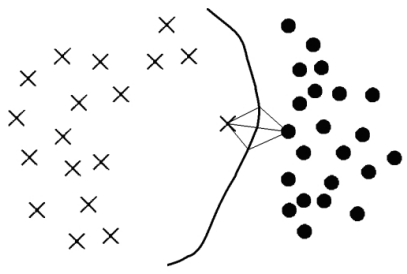

(b)

Fig. 1. A two-class separation problem is presented: (a) linear separation performed by SVM-based classifier cannot yield optimal solution in all cases; (b) distance of an object from the non-linear separation barrier should depend on the topology of the classes

the two closest $\times$ and $\bullet$ have the same distance from the non-linear separation barrier, but the $\times$ has better topology (the adjacent calculated distances are shorter for $\times$ than for the $\bullet$ point) so is considered closer than the $\bullet$ point.

\subsection{Study Records}

The BSPM signal resource contains 192-channels sampled at $1000 \mathrm{~Hz}$ with 12 bit resolution, obtained from the Research Institute for Technical Physics and Materials Science (MTA-MFA) of Budapest. These registrations, which were separated in two independent series, hold various malfunction cases, such as WPW syndrome, atrial and ventricular fibrillation, flutter. Our 12-lead ECG registrations were recorded at the County Medical Clinic Nr. 4 of Târgu Mureş. These signals were sampled at $500-1000 \mathrm{~Hz}$ with 12 -bit resolution.

The coarse calibration of the implemented models, such as cell model, tissue model, torso model and spatial heart model was realized using the Series 1 BSPM measurements. The preliminary ECG analyzer system (PAS) uses both the Series 2 of the BSPM recods and our 12-lead registrations.

\subsection{The Approach of ECG Inverse Problem}

Most fundamental problems in theoretical ECG can be described by an inverse solution. Their goal is to describe bioelectric cardiac sources based on knowledge of the ECG and the volume conductor properties that surrounds the sources.

As mentioned earlier, the most cumbersome point of the inverse solutions consist in its stability. In order to decrease the sensibility of our solution, in our approach the heart model parameters are obtained indirectly. This approximation of the inverse problem is in contrast to methods that directly solve the matrix equation linking electrical sources with electrical potential fields to estimate the inverse ECG solution.

We constructed the PAS based on detailed, a priori knowledge of human anatomy and physiology. It was developed using an ANN, tested and validated by physicians in clinical environment (see Fig. 2). 
The most important parameter that describes a whole cardiac cycle is related to the site of origin of cardiac activation. This information was obtained using the PAS module, where the output of the ANN provides the initial heart model parameters.

The ECG generator unit uses the cell, tissue and torso model to simulate a BSPM or 12-lead ECG. The objective functions that assess the similarity between the measured and simulated signals were also determined. As the structure of the used models was determined by the anticipative general model creation unit (AGMCU), only the proper parameter values has to be established. These heart model parameters were determined and adjusted with the aid of optimization algorithms or in certain cases by physicians. The simulation procedure is performed until the objective functions satisfy the a priori given convergence criteria. Finally the parameters are validated by physicians.

\subsection{ANN-Based Preliminary ECG Analyzer System}

The high number of heart model parameters implies a high dimensional searching problem. The a priori biological knowledge must be used to drastically reduce the number of necessary dimensions of the parameter space of heart model. The role of PAS unit consists in a rough determination of the cardiac status and state, that is used to initialize the model parameters and to simplify the searching problem for the optimization system.

In the present study, the PAS was developed using a three-layer UNN. This network is capable of mapping the non-linear input-output relationship, with the desired degree of accuracy. An adaptively weighted coefficient calculation method was used to train the ANN. The input layer incorporates 192 neurons, corresponding to the number of body surface electrodes used in the present simulation study. In case of 12-lead records, the unused channels' signals were estimated. From heuristical considerations, the number of hidden layer neurons was selected to 125 . The output layer had 512 neurons, which corresponded to 32 ventricular myocardial segments of computer heart model. Sixteen cardiac cellular units were selected for each of the 32 myocardial segments in the ventricles, and each of these $16 \times 32=512$ sites was then paced in the forward simulation using the computer heart-torso model, generating the data set for training the ANN.

\section{$3 \quad$ Results}

A parameter classification algorithm was applied to distinguish normal QRS complexes from abnormal ones, in order to determine the specific differences between the normal and abnormal parameter values. For normal cases the detection ratio is practically $100 \%$. The signals presented in Fig. 3 were obtained via simulation using the initial parameter set for a normal and abnormal (bypass tract) situation.

Figure 4 presents a series of cell activation simulations. In Fig. 4(a) and (b) the simulation of the cell depolarization mechanism is presented. The excitation rises 


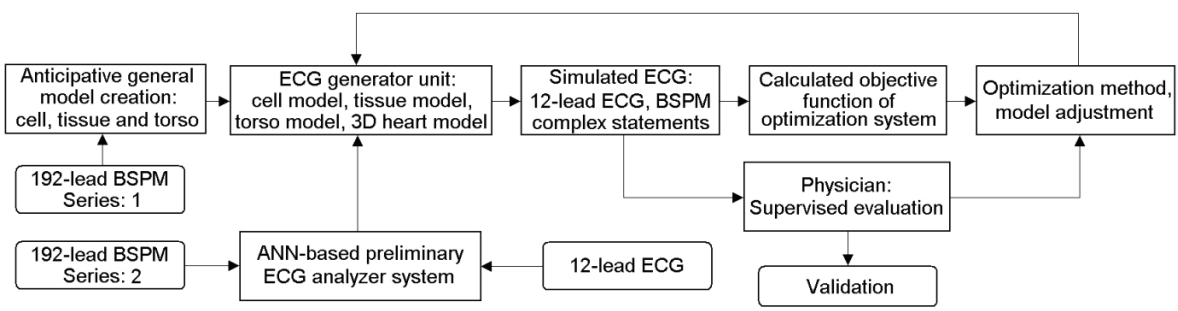

Fig. 2. The schematic diagram of the heart analyzer and modeling method

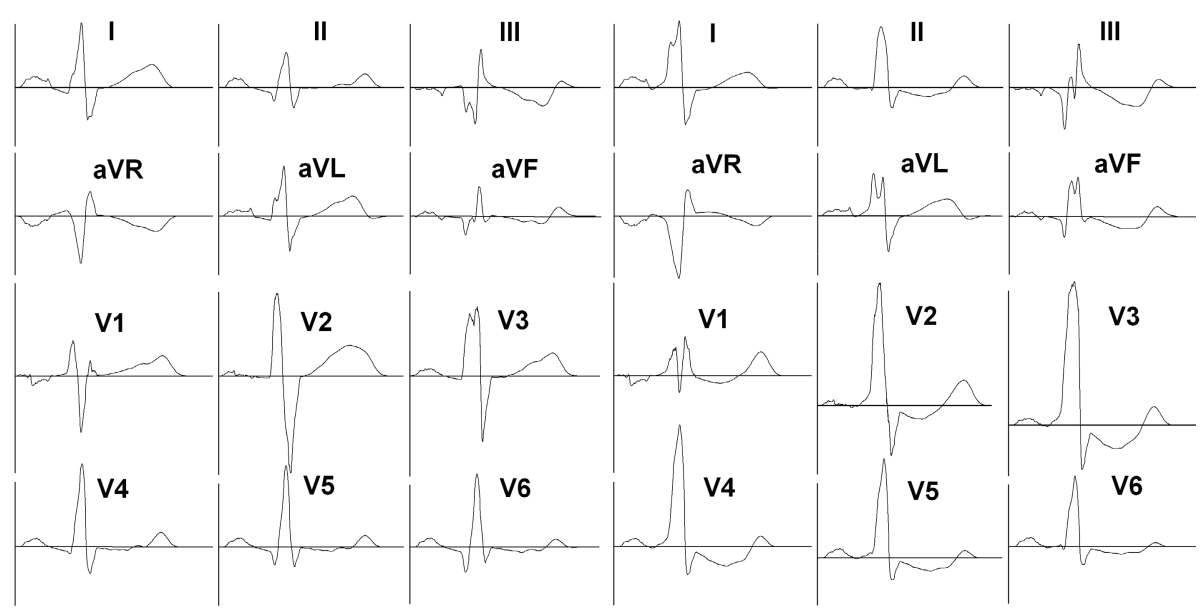

Fig. 3. The simulated ECG signal in normal and abnormal case (bypass tract)

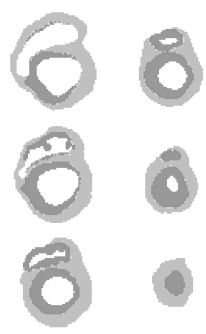

(a)

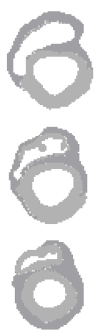

(b)
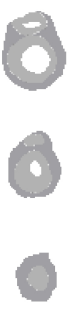

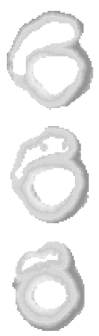

(c)

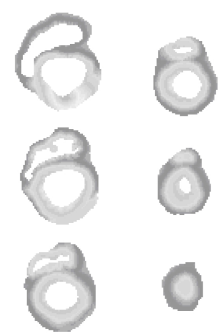

(d)

Fig. 4. The simulated depolarization in normal and abnormal case (bypass tract): (a) activated cells in normal case after $200 \mathrm{~ms}$ (b) activated cells in case of WPW syndrome after 200ms (c) activation time of cells in normal case (d) activation time of cells in case of WPW syndrome 
at the sino-atrial node. The state of the cells is illustrated with a 200ms delay from the excitation moment. Figures 4 (c) and (d) show the activation moment of the cardiac cells. The neighbor slices have $10 \mathrm{~mm}$ distance from each other, so a totally 5 centimeter wide ventricular tissue is visualized. The position of the slices is considered at a repolarized moment. Table 1 shows the efficiency of simulation for different cases. The evaluation of the simulated results was made by physicians. The performance was determined as the ratio of correct and total decisions.

Table 1. Simulation performance for normal and pathological cases

\begin{tabular}{lllr}
\hline Pathological case & $\begin{array}{l}\text { Correct } \\
\text { decisions }\end{array}$ & $\begin{array}{l}\text { Failed } \\
\text { decisions }\end{array}$ & Performance \\
\hline Normal & 49 & 0 & $100.00 \%$ \\
Ectopic beat & 24 & 0 & $100.00 \%$ \\
WPW syndrome & 15 & 1 & $93.75 \%$ \\
Atrial flutter & 43 & 2 & $95.55 \%$ \\
Atrial fibrillation & 20 & 1 & $95.23 \%$ \\
Ventricular fibrillation & 21 & 1 & $95.45 \%$ \\
Re-entry mechanisms & 29 & 3 & $90.62 \%$ \\
Triggered activity & 41 & 2 & $95.34 \%$ \\
Aberrant ventricular conduction & 21 & 1 & $95.45 \%$ \\
\hline Total cases & 263 & 11 & $95.98 \%$ \\
\hline
\end{tabular}

\section{Discussion and Conclusion}

Table 1 reveals that the 3D heart simulation 1718 succeeds in most cases, such as WPW (Wolf Parkinson White) syndrome, pre-excitations, and tissue activation modeling. The performance in case of re-entry mechanisms and triggered events is slightly decreased due to the hazard nature of the events. The application in practice of the model has several obstacles, which can be classified into the following groups:

- Effects of internal and external perturbations (such as environment, sympathetic and parasympathetic despondence);

- Lack of information on different elements of the model;

- Lack of technical background.

Several problems could be found, but the most important limitations are:

- The processes performed inside the cells are not well known, the behavior of the studied components cannot be determined with an acceptable precision;

- In critical cases, if a group of cells does not get the necessary food, it changes its behavior. A model created to simulate the normal behavior of the cell will not simulate it correctly in abnormal case; 
- Because the structure of the heart differs from patient to patient, this structure is not known a priori, it has to be determined in real-time, based on the available information;

- The structure of the torso introduces the same problem. It is hard to determine the electrical conductivity and precise position of its elements.

In case of human system identification the most important disturbing phenomena are:

- It is known, that respiration makes the heart change its shape and position. Although the motion of the heart can be tracked, it is not possible to determine from the ECG the amplitude of the motion;

- The continuous motion and displacement involves very hard problems. Because the motion has an effect on the behavior of all internal elements, the behavior of the heart will also be modified. The model has to follow the changes of the cell properties. For example: a resting man suddenly jumps out of the bed. The controlling mechanisms start their adjustment, the values of model parameters will change;

- Fever and respiration frequency can also cause alterations.

External events (the patient senses something annoying or pleasant) change the dependence between the previously measured signals, and the determined parameters. This is one of the causes why the perfect simulation of a human body is impossible.

At present, the performance of personal computers does not make possible the real-time determination of parameter values. The practical application is possible only in case of strongly parallel systems. The simplified model can be applied in real-time, but its efficiency is reduced because of the neglected parameters. The waveform of the simulated ECG in normal cases can be considered acceptable. The shape and duration of basic waves have realistic values. In case of abnormal cases the obtained waveform is not acceptable and more simulations are needed.

Acknowledgements. This research was supported by the Sapientia Institute for Research Programmes.

\section{References}

1. Cuppen, J.J.M., van Oosterom, A.: Model studies with inversely calculated isochrones of ventricular depolarization. IEEE Trans. Biomed. Eng. 31, 652-659 (1984)

2. Guanglin, L., Bin, H.: Localization of the site of origin of cardiac activation by means of a Heart-Model-Based electrocardiographic imaging approach. IEEE Trans. Biomed. Eng. 48, 660-669 (2001)

3. Haider, A.W., Larson, M.G., Benjamin, E.J., Levy, D.: Increased left ventricular mass and hypertrophy are associated with increased risk for sudden death. J. Am. Coll. Cardiol. 32, 1454-1459 (1998)

4. Johnson, C.R., MacLeod, R.S.: Adaptive local regularization methds for the inverse ECG problem. Progr. Biophys. Mol. Biol. 69, 405-423 (1998) 
5. Lagerholm, M., Peterson, C., Braccini, G., Edenbrandt, L., Sörnmo, L.: Clustering ECG complexes using Hermite functions and self-organizing maps. IEEE Trans. Biomed. Eng. 47, 838-848 (2000)

6. MacLeod, R.S., Brooks, D.H.: Recent progress in inverse problems in electrocardiology. IEEE EMBS Mag. 17(1), 73-83 (1998)

7. Minami, K., Nakajima, H., Yoyoshima, T.: Real time discrimination of the ventricular tachyarrhythmia with Fourier-transform neural network. IEEE Trans. Biomed. Eng. 46, 179-185 (1999)

8. Mirvis, D.M.: Validation of body surface electrocardiographic mapping. In: Mirvis, D.M. (ed.) Body surface electrocardiographic mapping, pp. 63-74. Kluwer, BostonDordrecht-London (1988)

9. Moreau-Villéger, V., Delingette, H., Sermesant, M., Ashikaga, H., McVeigh, E., Ayache, N.: Building maps of local apparent conductivity of the epicardium with a 2-D electrophysiological model of the heart. IEEE Trans. Biomed. Eng. 53, 14571466 (2006)

10. Noble, D.: Modeling the heart. Phys. 19, 191-197 (2004)

11. Osowski, S., Hoai, L.T.: ECG beat recognition using fuzzy hybrid neural network. IEEE Trans. Biomed. Eng. 48, 1265-1271 (2001)

12. Osowski, S., Hoai, L.T., Markiewicz, T.: Support vector machine-based expert system for reliable heartbeat recognition. IEEE Trans. Biomed. Eng. 51, 582-589 (2004)

13. Sermesant, M., Delingette, H., Ayache, N.: An electromechanical model of the heart for image analysis and simulation. IEEE Trans. Med. Imag. 25, 612-625 (2006)

14. Sermesant, M., Moireau, P., Camara, O., Sainte-Marie, J., Andriantsimiavona, R., Cimrman, R., Hill, D.L.G., Chapelle, D., Razavi, R.: Cardiac function estimation from MRI using a heart model and data assimilation: advances and difficulties. Med. Imag. Anal. 10, 642-656 (2006)

15. Shahidi, A.V., Savard, P., Nadeau, R.: Forward and inverse problems of electrocardiography: modeling and recovery of epicardial potentials in humans. IEEE Trans. Biomed. Eng. 41, 249-256 (1994)

16. Smola, A., Scholkopf, B.: A tutorial on support vector regression. Royal Holloway College, Univ. London, NeuroColt Tech. Rep. NV2-TR-1998-030

17. Szilágyi, S.M., Benyó, Z., Dávid, L.: Heart model based ECG signal processing. In: Proc. 5th IFAC Symp. Modell. Contr. Biomed. Syst. pp. 213-217 (2003)

18. Szilágyi, S.M., Benyó, Z., Dávid, L.: WPW syndrome identification and classification using ECG analysis. Proc. World Congr. Med. Phys. Biomed. Eng. 4423.pdf (2003)

19. Szilágyi, S.M.: Event recognition, separation and classification from ECG recordings. In: Proc. 20th Ann. Int. Conf. IEEE EMBS, pp. 236-239 (1998)

20. Thaker, N.V., Ferrero, J.M.: Electrophysiologic models of heart cells and cell networks. IEEE EMBS Mag. 17(5), 73-83 (1998)

21. Vapnik, V.: Statistical learning theory. Wiley, New York (1998) 